\title{
Induction of Caspase-Mediated Apoptosis in HepG2 Liver Carcinoma Cells Using Mutagen-Antioxidant Conjugated Self-Assembled Novel Carbazole Nanoparticles and In Silico Modeling Studies
}

Krishnan Anand,* Naeem Sheik Abdul, Terisha Ghazi, Muthusamy Ramesh, Gaurav Gupta, Murtaza M. Tambuwala, Harish Dureja, Sachin Kumar Singh, Dinesh Kumar Chellappan, Kamal Dua, Boomi Pandi, Muthupandian Saravanan,* and Anil Amichund Chuturgoon*

Cite This: ACS Omega 2021, 6, 265-277

Read Online

ABSTRACT: In this study, novel self-assembled carbazole-thiooctanoic acid nanoparticles (CTNs) were synthesized from amino carbazole (a mutagen) and thiooctanoic acid (an antioxidant). The nanoparticles were characterized using hyperspectral techniques. Then, the antiproliferative potential of CTNs was determined in HepG2 liver carcinoma cells. This study employed a solvent-antisolvent interaction method to synthesize a spherical CTN of size less than $50 \mathrm{~nm}$. Moreover, CT was subsequently capped to gold nanoparticles (AuNPs) in the additional comparative studies. The CT derivative was synthesized from carbazole and lipoic acid by the amide bond formation reaction using a coupling agent. Furthermore, it was characterized using infrared (IR), ${ }^{1} \mathrm{H}$ nuclear magnetic resonance, dynamic light scattering (DLS), and transmission electron microscopy techniques. The CTcapped gold nanoparticles (CTAuNPs) were prepared from CT, chloroauric acid, and $\mathrm{NaBH}_{4}$. The CTAuNPs were characterized using ultraviolet-visible, high-resolution TEM, DLS, and Fourier transform IR techniques. The cytotoxicity and apoptosis-inducing ability of both nanoparticles were determined in HepG2 cells. The results demonstrate that CTNs exhibit antiproliferative activity in the cancerous HepG2 cells. Moreover, molecular docking and molecular dynamics studies were conducted to explore the therapeutic potential of CT against human EGFR suppressor protein to gain more insights into the binding mode of the CT, which may show a significant role in anticancer therapy.

\section{INTRODUCTION}

Chemotherapeutic agents play a vital role in the treatment of cancer. Among them, carbazoles (a model DNA intercalator) and their derivatives have potential biological activities. ${ }^{1}$ The enhanced cellular internalization of carbazole and its reduced toxicity to normal cells are significant in biological studies. In the recent past, nanodrug-based strategies are widely used to combat multidrug resistance. ${ }^{2}$ The enhanced permeability and retention effect of self-assembled nanoparticles has garnered significant interest in drug delivery. A compound that combines two different drugs in one molecule has shown a synergistic effect in the treatment of diseases, and it can produce enhanced pharmacological effects. Such compounds are referred to as twin drugs and often show two different pharmacological activities in cancer cells. ${ }^{3}$ Although several metals are used for nanosynthesis, gold (inert metal) is preferred in medicine because of its low toxicity in healthy human cells. In general, antibodies and targeting moieties are conjugated by adsorption to the gold surface. A drawback of surface adsorption is the susceptibility of proteins to denaturation and in some cases limited ligand interactions with cell surface targets due to steric hindrance. ${ }^{4}$ The capping of organic ligands such as amines, thiols, ${ }^{5}$ dithiols, and so forth adds stability to gold nanoparticles. The interaction between a ligand and a nanometal has been investigated earlier. ${ }^{6}$ Dithiol ligands were conjugated to gold nanoparticles by sulfur ends. ${ }^{7}$ The organic-capping layer formed during metal-ligand interactions plays a vital role in high-performance biomaterials. ${ }^{8}$ Lipoic acid (LA) is a vitamin-like bioactive small molecule and is an antioxidant. The antioxidant exhibits important therapeutic potential under conditions where oxidative stress (ROS) is involved. It is a sulfur-rich compound found in cruciferous vegetables like broccoli and cabbage. ${ }^{9}$

Self-assembled nanoparticle-based drug-delivery systems are the most important for cancer chemotherapy and they are considered a better system because of their stability in the bloodstream, high drug loading, and controllable release from

Received: September 11, 2020

Accepted: December 9, 2020

Published: December 21, 2020 
carriers at target sites. ${ }^{10}$ Moreover, this strategy can improve the efficiency of cellular uptake because of their ability to enhance drug-delivery efficacy and reduce drug side effects. Different amphiphilic drug-drug conjugates have been made using dissimilar hydrophobic and hydrophilic drugs (Figure 1).

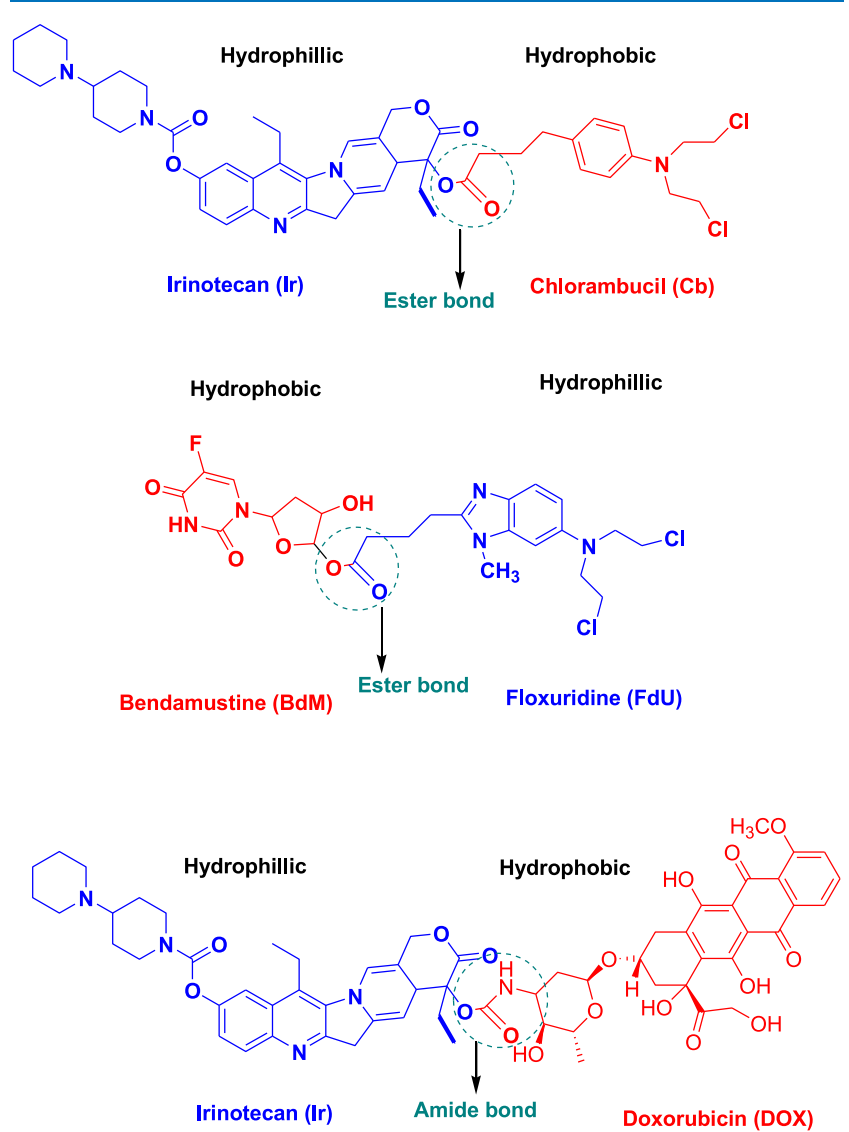

Figure 1. Literature-reported amphiphilic drug-drug conjugate for cancer therapy.

The resulting amphiphilic twin drugs could self-assemble into nanoparticles with high drug loading and improve cancer therapeutic efficacy. For example, irinotecan as a hydrophilic anticancer drug and a chlorambucil hydrophobic anticancer drug were conjugated through the hydrolyzable ester linkage. ${ }^{11}$ The amphiphilic nanoparticles were composed of two drugdrug conjugates, including doxorubicin (DOX)-chlorambucil $(\mathrm{Cb})$ and irinotecan $(\mathrm{Ir})-\mathrm{Cb}$ conjugates. Floxuridine $(\mathrm{FdU})$ as a hydrophilic anticancer drug was tethered with a hydrophobic anticancer drug of bendamustine to form an amphiphilic twin drug. ${ }^{12}$ The twin drug molecules interconnected by an ester bond or an amide bond could readily self-assemble into stable and uniform nanoparticles. The nanoparticles can be delivered to the action sites of a body via physical entrapment or chemical conjugation and better therapeutic efficacy against tumors without side effects over free drugs can be achieved. More importantly, after uptake by tumor cells and chemoenzymatic activity, the conjugates could be easily disintegrated into individual free drugs, which can induce nonoverlapping but synergistic pharmacological effects and simultaneously improve the therapeutic efficacy in vitro. Direct conjugation of hydrophobic drugs and small organic compounds is recently established as a new nano-drug delivery system. Because of a wide variety of therapeutic applications, nitrogen-containing heterocycles hold their significance in medicine, ${ }^{13}$ and carbazole derivatives are one such example. The thiooctanoic acid (lipoic acid) belongs to the family of tocopherols and tocotrienols. The mutagen, amino carbazole (AC) and the antioxidant, lipoic acid (LA) are the unique compounds; they are expected to excel for cancer therapeutic applications and may be suitable candidates to solve the drawbacks. In the present study, the hydrophobic AC and hydrophilic LA were chosen for achieving a synergistic combination in chemotherapy. Moreover, carbazole thiooctanoic acid (CT)-functionalized gold nanoparticles were synthesized. The newly synthesized carbazole self-assembled nanoparticles and conjugated gold nanoparticles were evaluated for their antiproliferative activities against HepG2 cells. The amphiphilic self-assembled nanoparticles (CTN) increased the activity of the extrinsic caspase 8 , intrinsic caspase 9, and executioner caspases and LDH release was not altered significantly suggesting apoptosis instead of necrosis. Furthermore, the molecular docking and molecular dynamics of mono CT molecules to epidermal growth factor receptor (EGFR) were studied, to explore the other possible targets.

\section{RESULTS AND DISCUSSION}

The compound CT [3] was synthesized by reacting 3-amino9-ethyl carbazole (AC) [1] and LA [2] in the presence of HBTU and DIEA. The base deprotonates the carboxylic acid. The resulting carboxylate anion attacks the electron-deficient carbon atom of HBTU (Figure 2). The resulting HOBt anion reacts with the newly formed activated carboxylic acid-derived intermediate to form an OBt-activated ester. The amine reacts with the OBt-activated ester to form the amide product amphiphilic CT.

Lipoic acid is a water-soluble antioxidant and AC is a waterinsoluble DNA intercalator. Therefore, the resulting CT molecule is amphiphilic and self-assembles to form nanoparticles in an aqueous environment, a benefit derived from the amphiphilic nature (Figure 3). The dialysis method was employed to prepare the self-assembled CT twin drug nanoparticles. The acetone solution of CT was added with deionized water and dialyzed to remove the acetone, thereby a stable nanoparticle solution was obtained with $0.5 \mathrm{mg} / \mathrm{mL}$ concentration.

The characteristic amphiphilicity of the CT provides a chance for itself to self-assemble into organic nanoparticles in water. The transmission electron microscopy (TEM) study determines the size and morphology of the self-assembled nanoparticles (scale bars: 200 and $50 \mathrm{~nm}$ ) (Figure 4). The TEM image shows that the CT nanoparticles aggregate into approximate spherical particles in aqueous solution, and the size determined by TEM is about $70 \pm 8.0 \mathrm{~nm}$, The DLS results in (Figure 5) show that the CT nanoparticle solution forms aggregates and the mean hydrodynamic diameter of CT nanoparticle aggregates is about $371.5 \mathrm{~nm}$ with a narrow unimodal size distribution. This size is smaller than that measured by DLS because of the shrinkage of CT nanoparticles in a drying nonsolvated state during TEM sample preparation. The solution of CT nanoparticles was stored at 4 ${ }^{\circ} \mathrm{C}$ in a refrigerator. The value of PDI is always under 0.041 at room temperature (RT) (Figure 5). The results demonstrate that $\mathrm{CT}$ nanoparticles are extremely stable during storage.

The CT-capped gold nanoparticles (CTAuNPs) were formed by the addition of an aqueous solution of CT to the chloroauric acid solution. After stirring the solution at RT, 

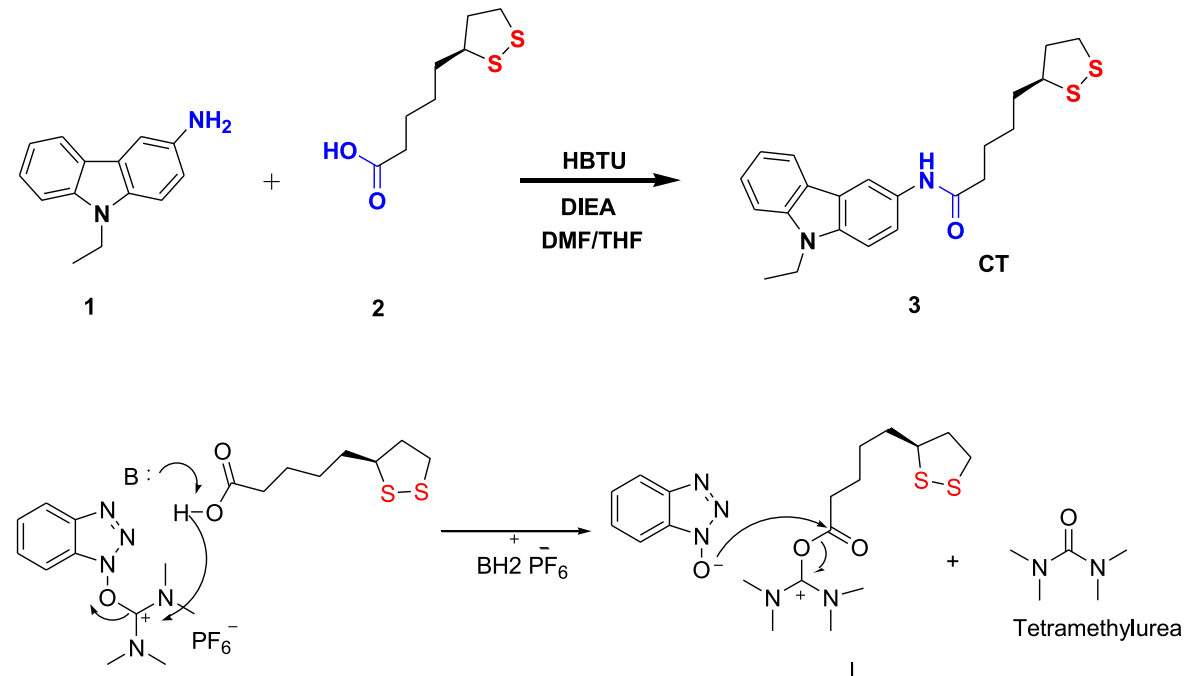

HBTU
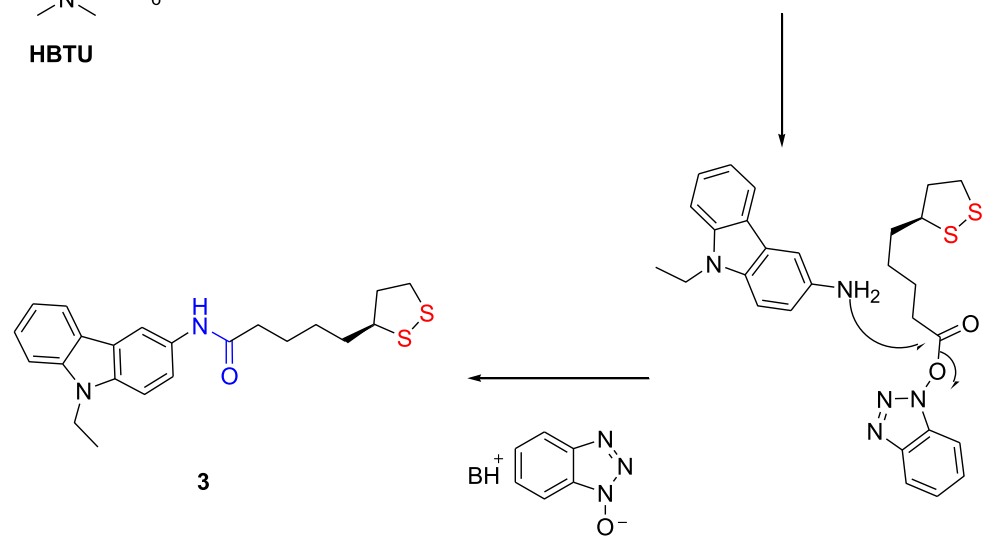

Figure 2. Synthesis of CT [3] and the plausible "amine to amide" mechanism for the formation of CT.

$\mathrm{NaBH}_{4}$ was added dropwise. Figure 6 shows the synthesis of CTAuNPs.

The formation of gold nanoparticles was initially confirmed when the solution turned into ruby red color. In Figure 7, the UV-vis spectra of CTAuNPs are shown. The characteristic peak at $530 \mathrm{~nm}$ (Figure 7 curve a) indicates the formation of gold nanoparticles, which was because of the surface plasmon excitation of gold nanoparticles. ${ }^{26}$ A bathochromic shift with the appearance of a broad peak at $552 \mathrm{~nm}$ (Figure 7 curve b) was observed because of the aggregation and surface modification of gold nanoparticles. When carbazole interacted with the gold nanoparticle, the ruby red color rapidly changed into blue.

The UV-vis spectrum of the synthesized gold nanoparticles was obtained. The size and shape of the nanoparticles were observed with a transmission electron microscope. Zeta potential measurements were carried out using a dynamic light scattering instrument. The observed nanoparticles were monodispersed and exactly spherical or nearly spherical with size ranging from 5-10 nm (Figure 8). Also, spherical-shaped gold nanoparticles were observed.

Zeta potential is an indication of colloidal stability. ${ }^{27}$ Zeta potential of nanoparticles with $>+30$ or $<-30 \mathrm{mV}$ is more stable. Colloids having a lower zeta potential result in aggregation because of Van Der Waals forces. ${ }^{27}$ Different principles were adopted for the measurement of the particle size using HRTEM and DLS. Hence, the particle size measured by HRTEM and DLS differs marginally. Zeta potential was found to be $-0.172 \mathrm{mV}$ (Figure 9B), which shows their least stability. The average hydrodynamic particle size obtained by DLS is $45 \mathrm{~nm}$ (Figure 9A), which is identical to that obtained by HRTEM.

Figure 10 shows the FTIR spectra, which compare CT and CTAuNPs. A stretching frequency at 3237 and $1587 \mathrm{~cm}^{-1}$ confirmed the $\mathrm{NH}$ and $\mathrm{S}-\mathrm{S}$ functional groups of CTN. The $\mathrm{NH}$ stretching band was observed for CTAuNPs, but it was shifted to higher values. Also, the sulfur bond present in CT was absent in CTAuNPs, thereby confirming an attachment of $\mathrm{CT}$ into the gold surface. The $\mathrm{S}-\mathrm{S}$ group was present in the IR spectra as indicated in Figure 10A,B, indicating that it did not participate in any linkage or interaction with the gold surface.

2.1. In Vitro Assays. The anticancer potential of CTN and CTAuNPs was determined using a liver carcinoma-derived (HepG2) cell line. The antiproliferative activities were screened using the MTT assay.

The decrease in cell viability after exposure for $6 \mathrm{~h}$ was dosedependent with higher concentrations displaying the most significant loss to cell viability. $\mathrm{IC}_{50}$ values obtained for CTN and CTAuNPs were 91.3 and $432 \mu \mathrm{g} / \mathrm{mL}$, respectively (Figure 11). While gold-capped nanoparticles easily penetrate cell membranes and are often described as an effective drug carrier, our data indicate that the self-assembled organic CTNs displayed greater effectiveness in decreasing cell viability when compared to the gold derivative. Gold nanoparticles display diverse and unique properties that may contribute to cell protective mechanisms after acute treatments such as antioxidant defense mechanisms and altered the energy flux. 


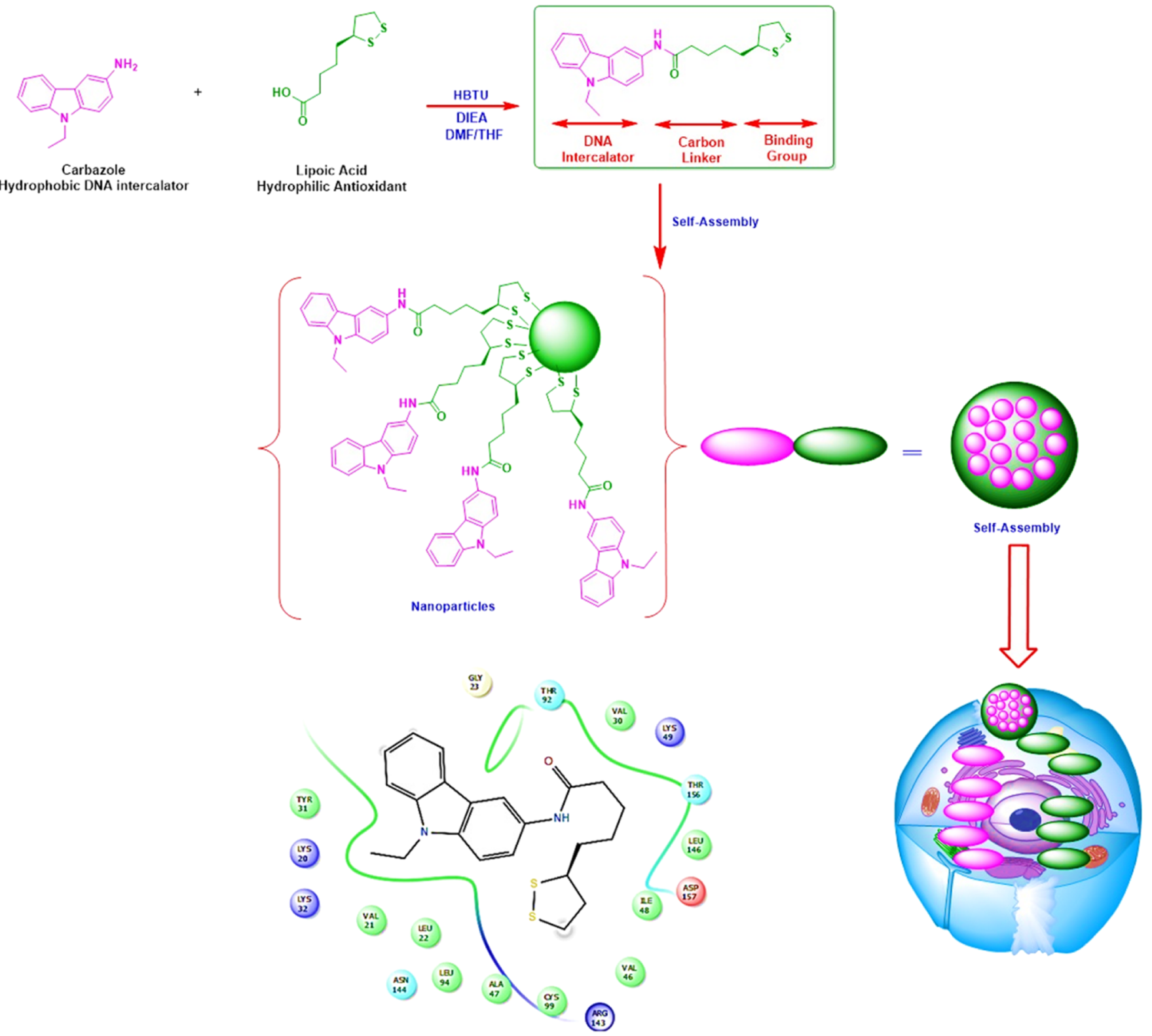

Figure 3. Schematic route for the formation of an amphiphilic twin bioactive molecule and its self-assembly for endocytosis.

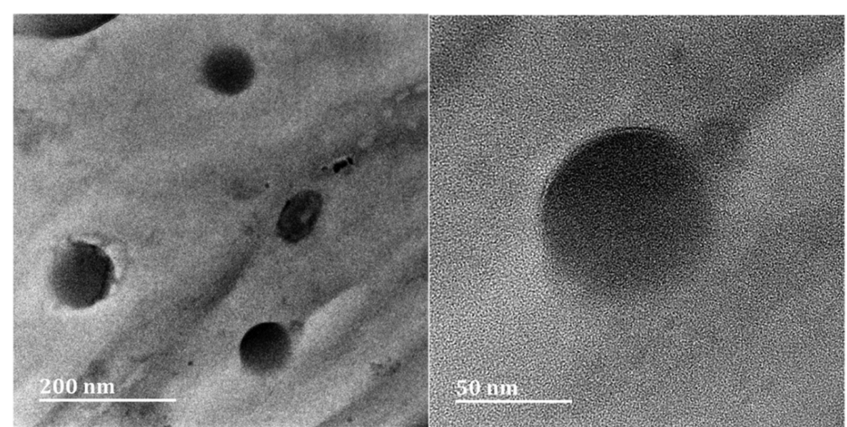

Figure 4. Morphology of amphiphilic CT nanoparticles.

Therefore, only CTN was selected for further biological assessment.

The cytotoxic potential of carbazole derivatives has already been estimated using several in vitro models. ${ }^{28}$ Our innovative conjugation of a carbazole to an antioxidant and a mitochondrial stimulator, that is, $\alpha$-LA, has shown a profound effect on caspase initiation and activation (a marker for apoptosis). Apoptosis or programmed cell death regulates the elimination of damaged cells to maintain homeostasis. Caspases are critical facilitators of apoptosis as they initiate and execute the process via two pathways: the extrinsic and the intrinsic pathways. The extrinsic pathway is stimulated by ligands binding to receptors that regulate downstream adaptor molecules resulting in caspase 8 activation (Figure 12). The intrinsic pathway involves the binding of caspase 9 to the apoptotic protease-activating factor-1 (APAF-1) apoptosome complex in response to mitochondrial signals such as membrane depolarization. Both pathways result in the activation of executioner caspases, caspases 3/7.

Our data indicate the enhanced activity of executioner caspases $3 / 7$ (Figure 13C). The initiators of the extrinsic (Figure 13A) and intrinsic (Figure 13B) apoptotic pathways are also upregulated. It is intriguing that caspase activity increases in a dose-dependent manner but drastically decreases 


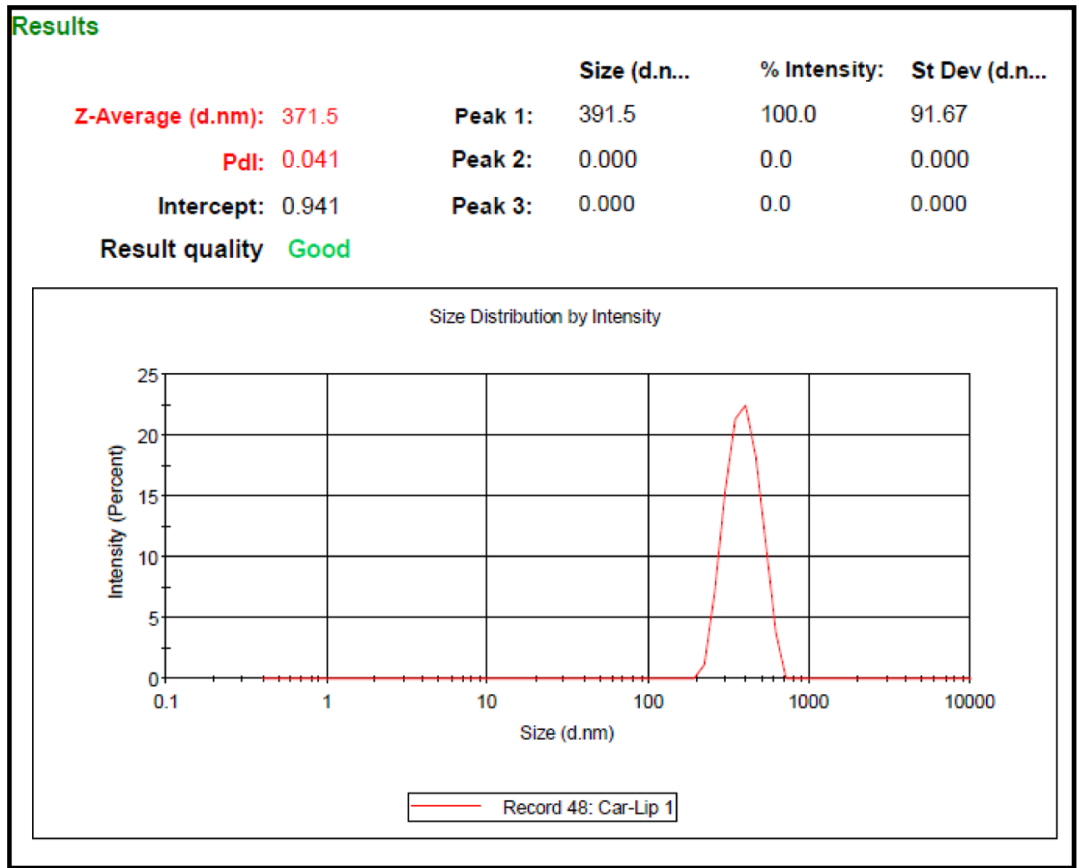

Figure 5. Dynamic light scattering (DLS) profile: size distribution of CT nanoparticles with PDI:0.041.
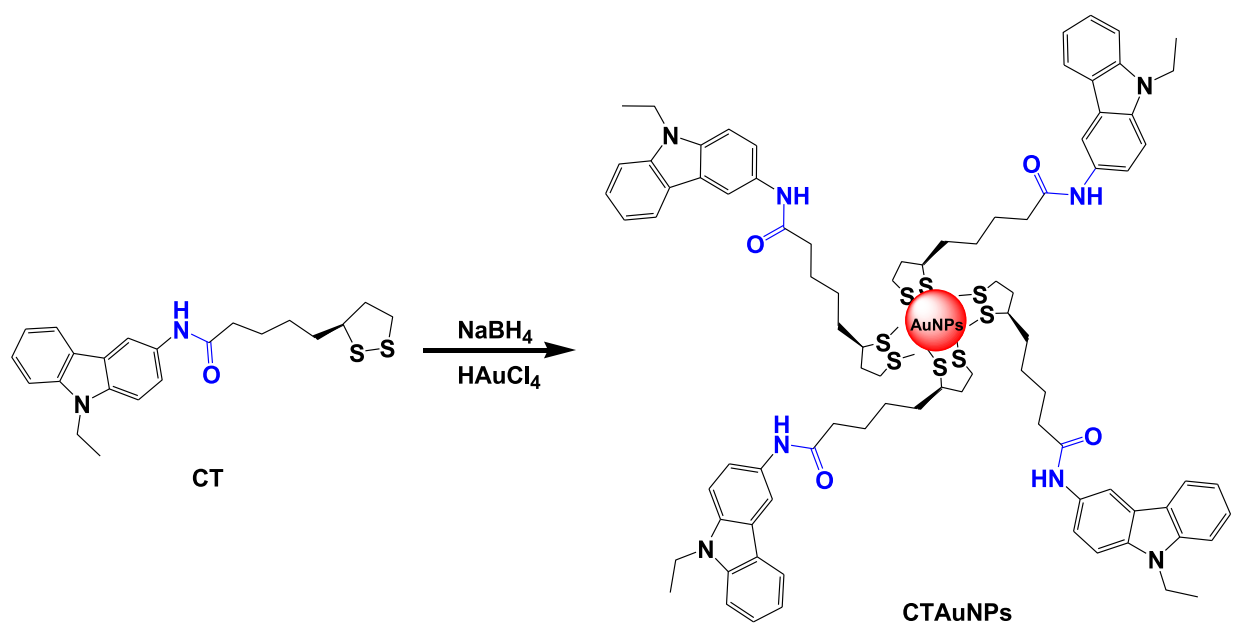

Figure 6. The outline for the synthesis of CTAuNPs.

at the highest CTN concentration. We speculate that this may be because of membrane receptor saturation and rapid ATP depletion (Figure 14).

The plasma membrane integrity was evaluated by determining $\mathrm{LDH}$ release into the supernatant. Plasma membrane leakage is strongly correlated with overt cytotoxicity and necrotic cell death. We observed no significant changes to the levels of released LDH (Figure 15) suggesting that apoptosis was responsible for cell death and not necrosis. The results are in agreement with the caspase activity assays. Triggering apoptosis without overt necrosis would be the preferred means of destroying cancer cells as it dampens many of the damaging side effects. This is important to normal healthy cells that may not be affected by the drug and hence eliminate any unwanted side effects generally associated with chemotherapy.

2.2. In Silico Analysis to Investigate the Other Anticancer Target. Molecular docking and molecular dynamics are the in silico approaches to predict the ligandbinding pose inside the target protein or host molecule. ${ }^{29}$ The application of molecular docking to predict the therapeutic and metabolic profiles has been well documented. ${ }^{30}$ In the present study, molecular docking and molecular dynamics have been employed to investigate the therapeutic anticancer potential of carbazole, LA, and CT.

Molecular docking has been used as a tool to evaluate the interaction and geometric conformation of a ligand-biological target. $^{31}$ The potential mechanism of carbazole is the inhibition of EGFR. The EGFR is a validated target for the treatment of cancer. $^{28,32}$ Therefore, carbazole, LA, and CT were docked into the binding site of the EGFR to explore the anticancer therapeutic potential. Carbazole, LA, and CT were, respectively, shown the docking scores of $-7.2,-4.8$, and -7.9 $\mathrm{kcal} / \mathrm{mol}$ (Table 1 ). The range of root mean square deviation (rmsd) for each of the molecule [AC, LA, and CT] from the top ten ranked pose is shown in Table 1 . CT has shown the highest docking scores of $(-7.9 \mathrm{kcal} / \mathrm{mol})$ in comparison to carbazole and LA. CT is the combined molecular fragments of carbazole and LA. The combined molecular feature may be 


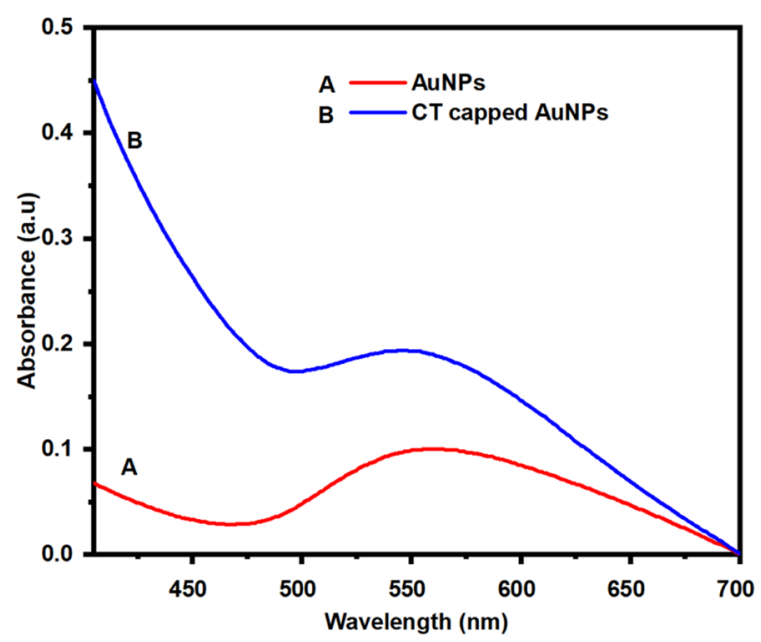

Figure 7. UV-vis spectra of gold nanoparticles, line (A) (red color) and CTAuNPs, line (B) (blue color).

responsible for the achievement of the highest molecular docking score. The interacting residues of EGFR and its interaction with mono CT are shown in Figures 16 and 17. Val21, Leu22, Ala47, Thr92, Asp157, and Arg143 were found to be binding site residues for $\mathrm{CT}$ at the binding site of the EGFR in molecular docking.

The highest docking score of $-7.9 \mathrm{kcal} / \mathrm{mol}$ has been obtained for the complex of CT with EGFR in molecular docking. Therefore, the complex has been subjected to molecular dynamics simulations. The results of molecular dynamics simulations help to ensure the binding affinity of the ligand and stability of the complex from the estimated values of energy components. Molecular dynamics simulation was carried out for the complex of CT with EGFR using AMBER $18 .^{33}$ Initially, the molecular docking complex was pretreated in Chimera before subjecting to molecular dynamics. ${ }^{34}$ The ligand-bound complex was used as an initial geometry for molecular dynamics. The ligand and the protein were parametrized in Antechamber and tleap. ${ }^{35}$ The complex was neutralized by adding $\left(\mathrm{Na}^{+} / \mathrm{Cl}^{-}\right)$as counter ions and the complex was solvated using the TIP3P water model. ${ }^{36}$ The minimization of the complex was carried out for 200 steps. The heating and equilibrium were conducted at $300 \mathrm{~K}$. Then, the simulation of the complex system was carried out for $5 \mathrm{~ns} .^{37}$ The results of the trajectories were saved for every 1 ps and were analyzed using the CPPTRAJ module. ${ }^{38}$ After the $5 \mathrm{~ns}$ simulation, the binding free energy of the ligand-protein complex (CT with the EGFR) was estimated using the molecular mechanics/Poisson Boltzmann surface area (MM/ PBSA) method. The estimated energy components are depicted in Table 2. The estimated VDWAALS components were found to be $-45.31 \mathrm{kcal} / \mathrm{mol}$. The stability and flexibility of the complex were analyzed from the RMSD and RMSF plot (Figure 18). Moreover, the complex has shown a strong binding free energy of $(-39.86 \mathrm{kcal} / \mathrm{mol})$ according to the binding free energy calculations (Table 2). This strong binding affinity of CT with EGFR shows its therapeutic potential as an anticancer agent.

\section{CONCLUSIONS}

In this study, self-assembled amphiphilic CTNs, that is, aminocarbazole (mutagen) and LA (antioxidant), were utilized

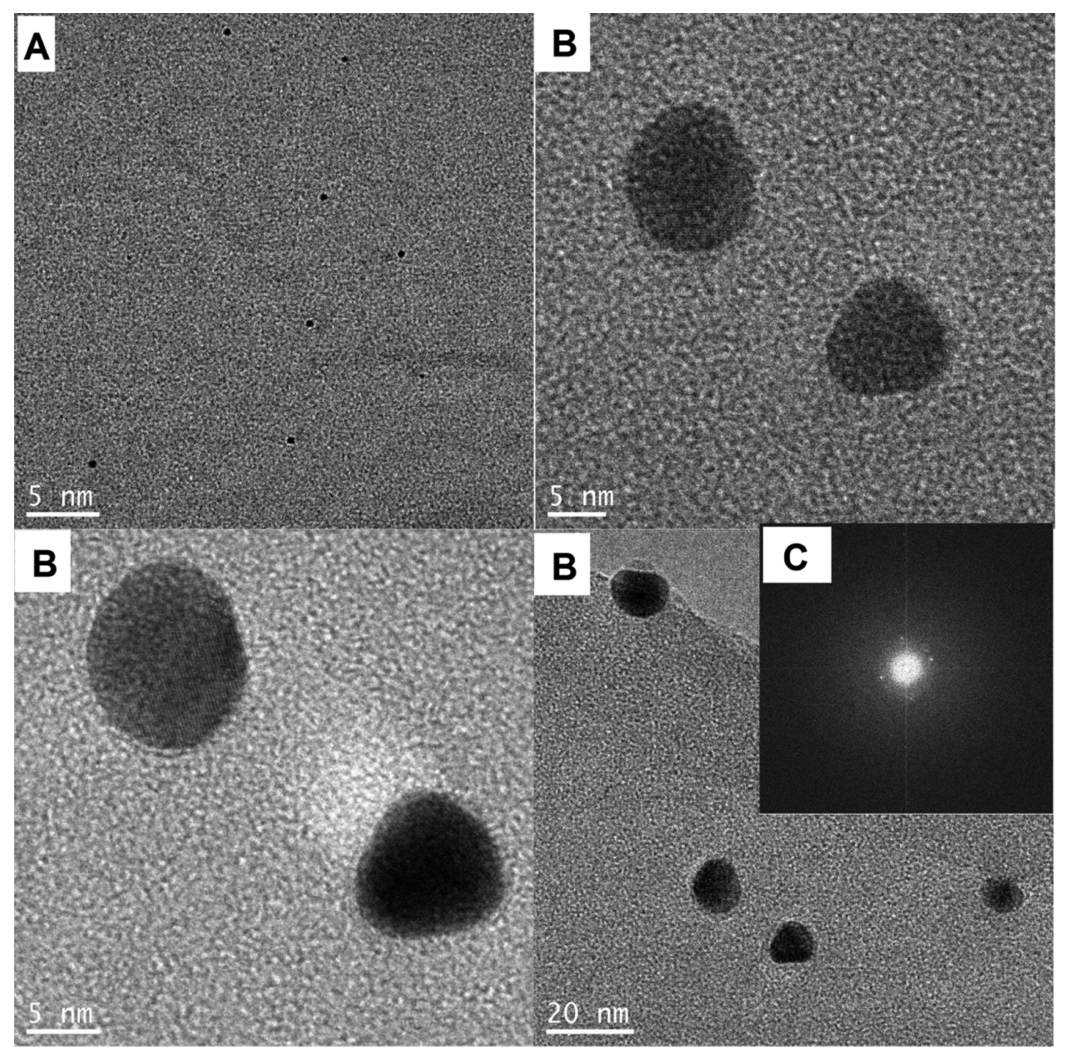

Figure 8. High-resolution TEM images of (A) gold nanoparticles, (B) gold nanoparticles capped with CT, and (C) part of spherical gold nanoparticles and their corresponding fast Fourier transformed image. 


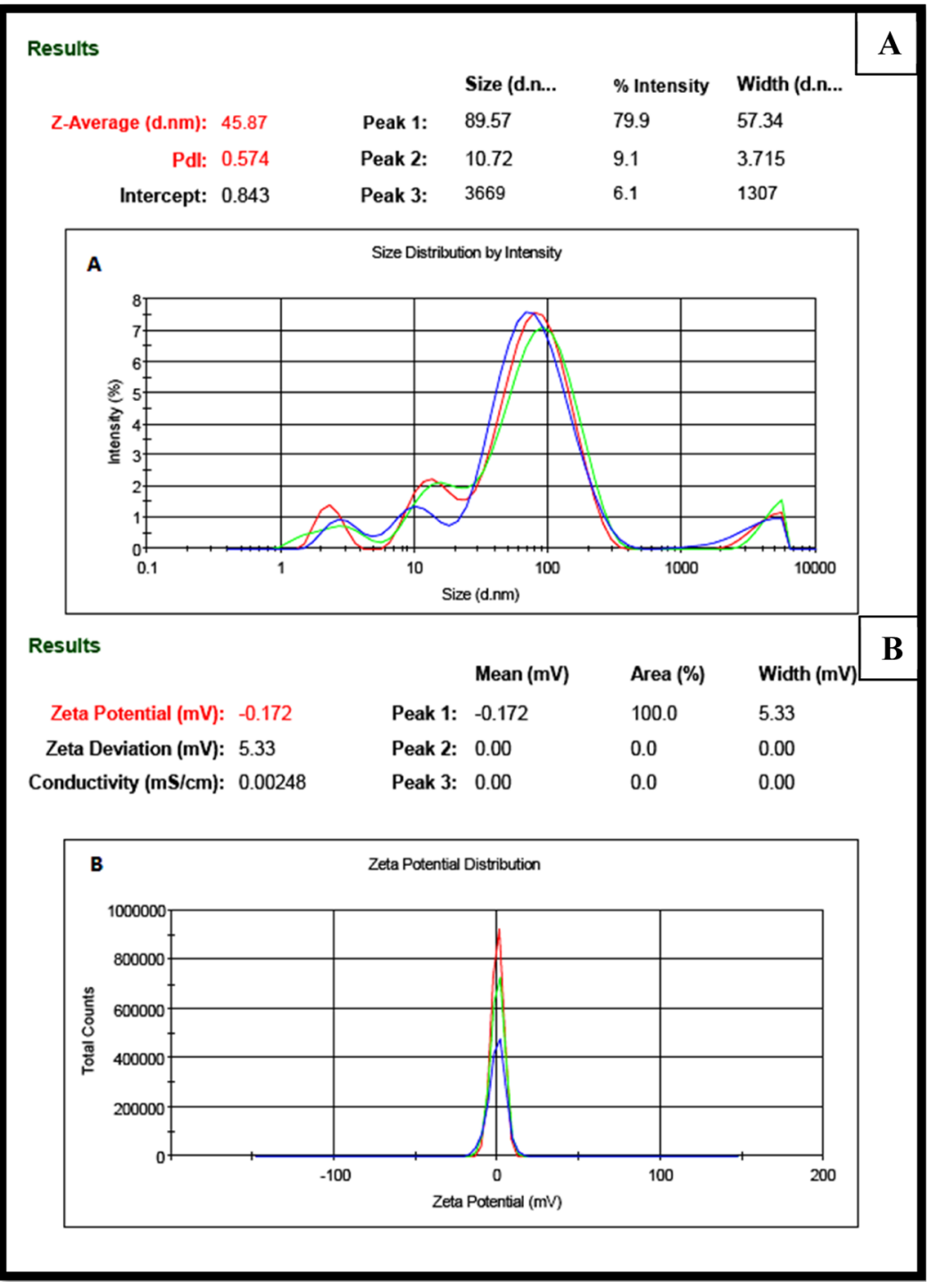

Figure 9. (A) Particle size distribution of CTAuNPs using the DLS method (B) Zeta potential measurement using a Zetasizer.

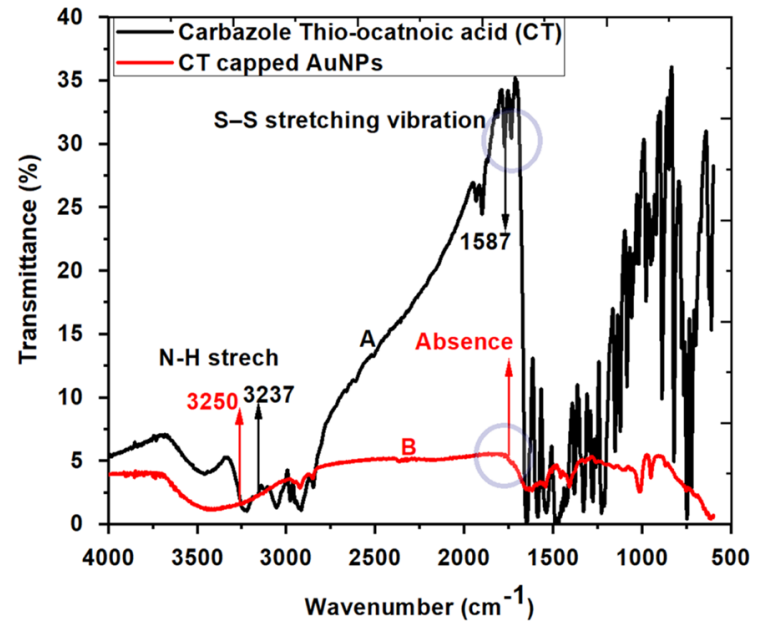

Figure 10. FTIR Profile of CT (line A) and CTAuNPs (line B).

as two in one molecule to investigate the biochemical mechanism of the binary molecules on human cancerous liver (HepG2) cells. The CTAuNPs were synthesized, characterized, and apoptotic induction activity of the same was studied. To prove the interaction between disulfide and AuNPs, the spectroscopic analysis was performed. It showed the disulfide group of carbazole LA acts as a potential site to conjugate with the gold surface at the nanoscale, resulting in carbazole-capped gold nanoparticles. The CTN increased the activity of the extrinsic caspase 8 , intrinsic caspase 9, and executioner caspases and $\mathrm{LDH}$ release was not altered significantly suggesting apoptosis instead of necrosis in liver carcinoma (HepG2) cells. The results indicated that selfassembled carbazole nanoparticles CTN induce apoptosis in the absence of overt necrosis in liver carcinoma (HepG2) cells and it may be a novel anticancer agent. Moreover, the in silico studies like molecular docking and molecular dynamics have shown the strong binding affinity for CT with EGFR. In postdynamics, this complex has shown substantial stability during the simulation. Therefore, CT may act as a potential anticancer agent.

\section{MATERIALS AND METHODS}

4.1. Chemicals and Reagents. Gold (III) chloride trihydrate $\left(\mathrm{HAuCl}_{4} \cdot 3 \mathrm{H}_{2} \mathrm{O}\right)$, 9-ethyl-3-AC, hexafluorophosphate benzotriazole tetramethyl uranium (HBTU), diisopro- 
A

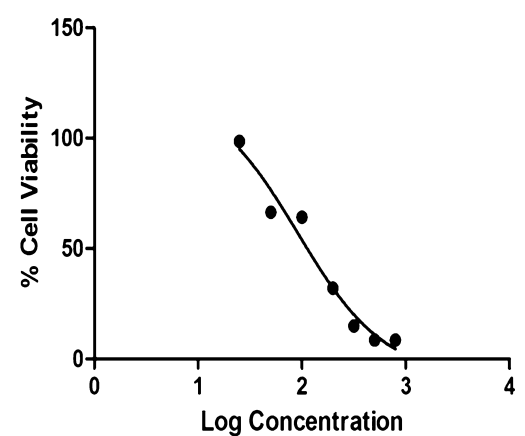

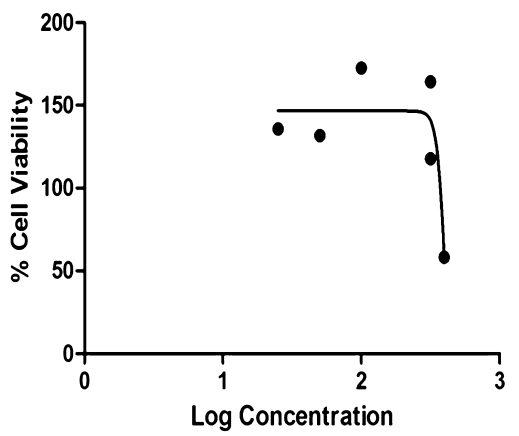

Figure 11. MTT evaluation of cell viability for CTN (A) and CTAuNPs (B) in HepG2 cells.

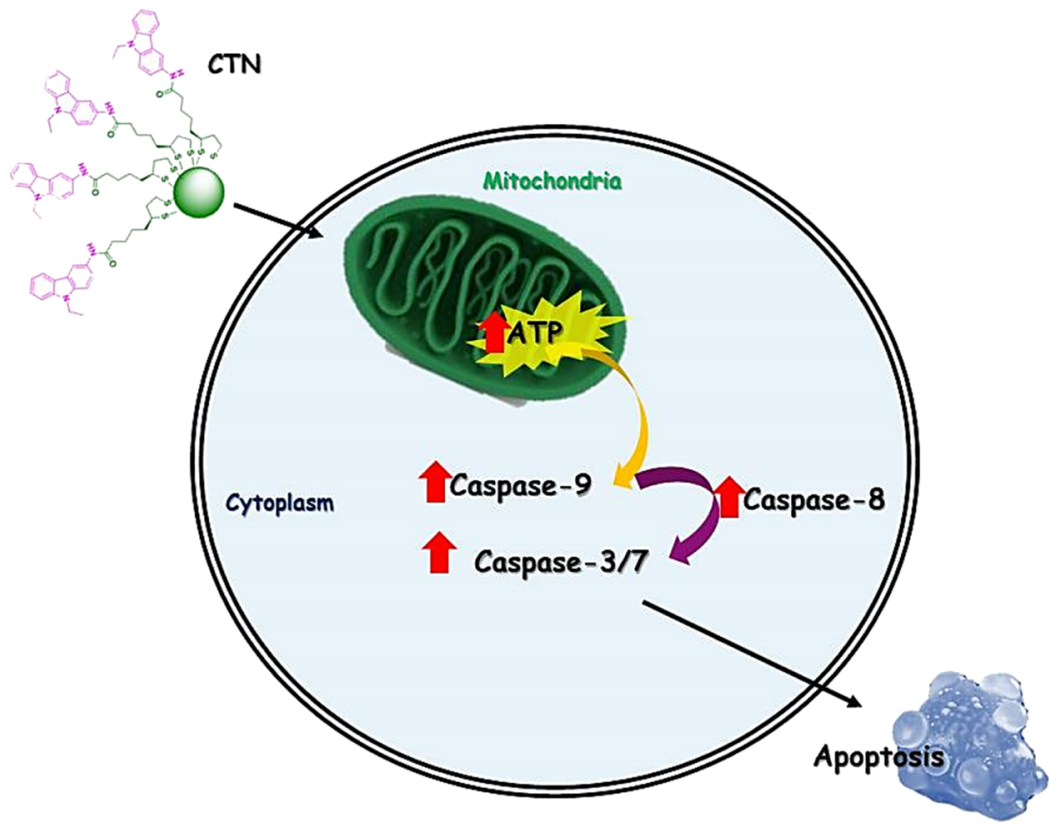

Figure 12. Schematic representation of CTN-induced apoptosis.
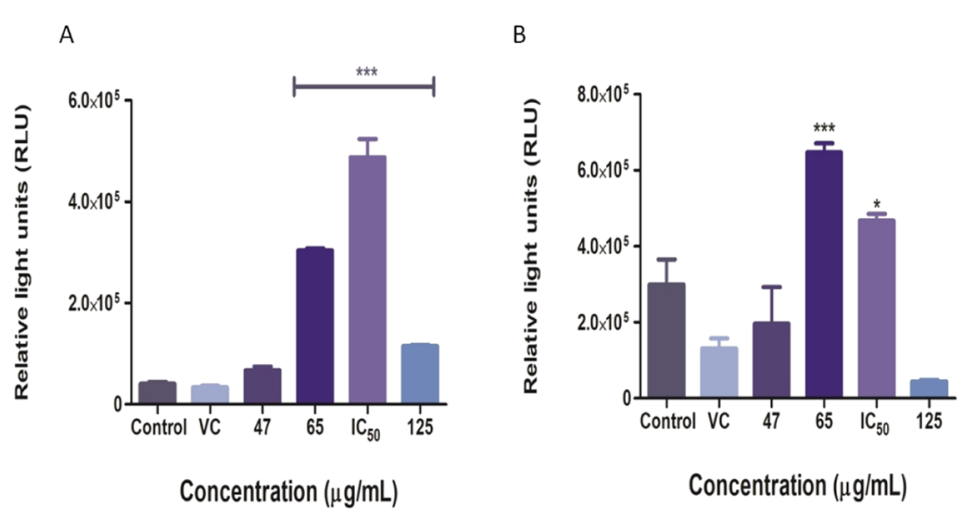

C

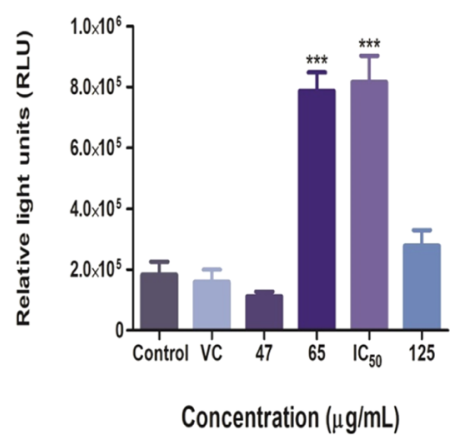

Figure 13. (A) CTN increased the activity of the extrinsic caspase 8, (B) intrinsic caspase 9, and (C) executioner caspases $3 / 7$. Key: $* * * p<0.001$ $p<0.05$.

pylethylamine (DIEA), LA, and $\mathrm{NaBH}_{4}$ were procured from Sigma-Aldrich, South Africa. Other chemicals were procured as analytical grade and do not require purification. Reagents used for the study were prepared using distilled water. Glassware was washed thoroughly using aqua regia followed by doubledistilled water.
4.2. Synthesis of Novel CT. $15 \mathrm{~mL}$ of dimethylformamide (DMF) and $5 \mathrm{~mL}$ of tetrahydrofuran (THF) were used to solubilize LA (0.55 g, $2.75 \mathrm{mmol})$ and then added with HBTU ( $1 \mathrm{~g}, 3.05 \mathrm{mmol})$, DIEA ( $1 \mathrm{~mL}, 6.00 \mathrm{mmol})$, and 9-ethyl-3-AC $(0.56 \mathrm{~g}, 2.7 \mathrm{mmol})$. The resulting mixture was fully dissolved using a magnetic stirrer at RT. TLC analysis was performed to check for conjugation. $50 \mathrm{~mL}$ of distilled water was added to 


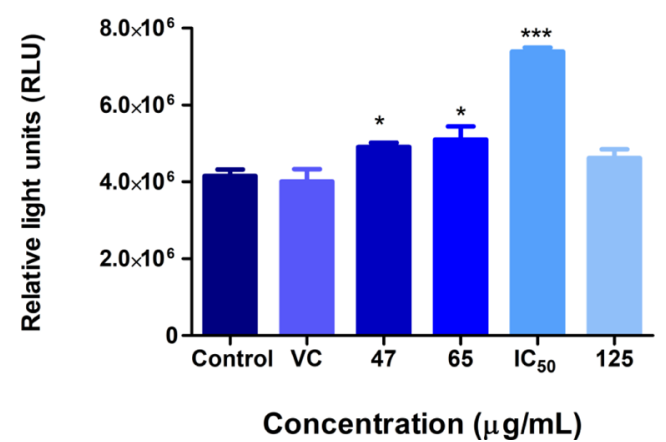

Figure 14. Effect of CTN on ATP detection.

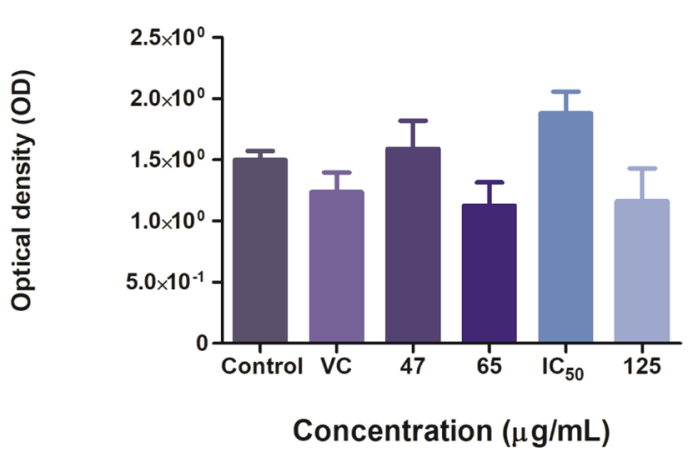

Figure 15. Effect of CTN on LDH leakage.

Table 1. Molecular Docking Scores of the Molecules (Aminocarbazole, Lipoic Acid, and CT)

\begin{tabular}{clclc} 
no. & \multicolumn{1}{c}{ molecule } & docking scores & RMSD (lb) & RMSD (ub) \\
1. & aminocarbazole & -7.2 & $0.00-1.598$ & $0.0-4.567$ \\
2. & LA & -4.8 & $0.00-4.479$ & $0.0-5.888$ \\
3. & CT & -7.9 & $0.0-3.202$ & $0.0-6.032$
\end{tabular}

the resulting mixture and then extracted with ethyl acetate $(25$ $\mathrm{mL}$ ) three times. The ethyl acetate layer was combined and then dried by passing it through anhydrous sodium sulfate followed by evaporation to yield a crude product. Finally, a white solid CT was obtained after purification using column chromatography (50:50 EtOAc/hexane). Yield: $1.10 \mathrm{~g}$ (92\%); mp: $120{ }^{\circ} \mathrm{C}$; IR $\left(\mathrm{KBr}, \mathrm{cm}^{-1}\right)$ : 3462.01, 3237.92, 3058.40, 2917.84, 2513.46, 2513.46, 1900.406, 1773.85, 1736.05, $1650.38,1587.43,1542.65,1485.11,1382.07,1277.01$, $1228.44,1152.46,1123.774,1085.725,1060.128,1020.12$, 1020.128, 978.56, 888.97, 821.95; ${ }^{1} \mathrm{H}$ NMR (400 MHz, $\left.\mathrm{CDCl}_{3}\right): \delta(\mathrm{ppm}) 1.35(\mathrm{~m}, 2 \mathrm{H}), 1.39(\mathrm{~s}, 3 \mathrm{H}), 1.56(\mathrm{q}, 2 \mathrm{H})$, $1.68(\mathrm{~m}, 2 \mathrm{H}), 1.80(\mathrm{~m}, 2 \mathrm{H}), 2.40(\mathrm{t}, 2 \mathrm{H}), 2.62(\mathrm{~m}, 1 \mathrm{H}), 3.34$ $(\mathrm{m}, 2 \mathrm{H}), 3.5(\mathrm{t}, 1 \mathrm{H}), 4.35(\mathrm{q}, 2 \mathrm{H}), 7.0-7.1(\mathrm{~s}, 1 \mathrm{H}), 7.16-7.19$ $(\mathrm{t}, \mathrm{H}), 7.29(\mathrm{~s}, 1 \mathrm{H}), 7.31 .(\mathrm{s}, 1 \mathrm{H}), 7.37-7.36(\mathrm{~d}, 1 \mathrm{H}), 7.418-$ $7.41(\mathrm{~m}, 1 \mathrm{H}), 8.20(\mathrm{~d}, 1 \mathrm{H}), 8.42(\mathrm{t}, 1 \mathrm{H}) ;{ }^{13} \mathrm{C}$ NMR $(400$ $\left.\mathrm{MHz}, \mathrm{CDCl}_{3}\right): \delta(\mathrm{ppm}) 171.00,140.44,137.23,129.61$, $125.85,123.02,122.75,120.66,119.49,118.73,112.91,108.53$, $108.45,58.43,40.28,48.48,37.39,37.37,34.69,33.94,28.93$, 25.43, 24.94, 13.80 .

4.3. Formation of Carbazole Nanoparticles. At RT, novel CT twin bioactive molecules $(25 \mathrm{mg})$ were dissolved in acetone $(20 \mathrm{~mL})$. All the prepared CT solutions were syringe filtered (pore size $0.22 \mu \mathrm{m}$ ). Subsequently, the antisolvent deionized water $(20 \mathrm{~mL})$ was added in drops to the solution and stirred gently for half an hour, and the nanoparticle was precipitated. This technique was termed as the antisolvent precipitation technique. The appearance of turbidity indicated the formation of amphiphile CTNs from CT twin bioactive molecules.

4.4. Characterization of Carbazole Nanoparticles. Particle size ( $z$-average diameter, $\mathrm{d} / \mathrm{nm})$, polydispersity index (PDI), Figure 5, and zeta potential of the precipitated nanoparticles were analyzed using DLS (Zetasizer Nano ZS, Malvern Instrument Ltd., UK) at $25{ }^{\circ} \mathrm{C}$. Particle size and shape of the nanoparticles were characterized by TEM, Figure 4. CTNNPs $(1 \mu \mathrm{L})$ were kept on formvar-coated grids, air-dried, and observed at $100 \mathrm{kV}$ for TEM (JEOL 1010 TEM using a Megaview III camera and iTEM software) studies.

4.5. Synthesis of Novel Carbazole Thiooctanoic AcidCapped Gold Nanoparticles. The sodium borohydride reduction method was adopted to synthesize gold nanoparticles. ${ }^{14}$ Briefly, $0.01 \mathrm{~g}$ of $\mathrm{NaBH}_{4}$ was employed to reduce tetrachloroauric acid $\left(10^{-4} \mathrm{M}\right)$ leading to the synthesis of gold nanoparticles of $5 \mathrm{~nm}$ in diameter. The resulting nanoparticle solution was ruby-red in color. Subsequently, $10^{-3} \mathrm{M}$ aqueous solution of CT was used as a capping agent for gold nanoparticles. Then, the solution was repeatedly centrifuged (10,000 rpm for $1 \mathrm{~h}$ ) to purify the CTAu NPs.

The absorption spectra $(200-800 \mathrm{~nm})$ of the capped gold nanoparticle solution were measured using an ultravioletvisible UV-vis spectrometer (Varian Cary-50 UV spectrophotometer linked to a TCC-240A Shimadzu heating vessel temperature-controlled cell holder). To analyze the size and shape, $1 \mu \mathrm{L}$ of the CTAu NPs was kept on formvar-coated grids, air-dried, and observed using a transmission electron microscope. For FTIR studies, CTAu NPs were purified by centrifugation $(10,000 \mathrm{rpm}$ for $10 \mathrm{~min})$ and the resulting pellet was washed thrice using distilled water $(20 \mathrm{~mL})$. Then, the FTIR spectra were recorded using a Varian 800 FTIR spectrophotometer. Particle size and zeta potential were measured using a DLS Malvern Zetasizer Nano ZS (Malvern Instruments Ltd, UK) Merck 2423 instrument.

4.6. Cell Culture. The HepG2 human liver carcinoma cells were grown in $25 \mathrm{~cm}^{3}$ culture flasks $\left(37{ }^{\circ} \mathrm{C}, 5 \% \mathrm{CO}_{2}\right)$ in complete culture media (CCM, Eagles Minimum Essential media, supplemented with $10 \%$ fetal calf serum, $1 \%$ Lglutamine, and $1 \%$ penicillin-streptomycin-fungizone) until obtaining $90 \%$ confluence. Then, the cells were harvested by trypsinization and used for the relevant assays.

4.7. Cell Viability. MTT assay was adopted to analyze the cell viability. HepG2 cells $(15,000$ cells/well) were seeded in a 96 well microtiter plate and incubated overnight to adhere to the plate. The cells were incubated for $6 \mathrm{~h}$ with varying concentrations of CTN and CTAu $(0-750 \mu \mathrm{g} / \mathrm{mL})$ in five replicates. The plate was incubated at $37{ }^{\circ} \mathrm{C}$ for $4 \mathrm{~h}$ after the addition of $120 \mu \mathrm{L}$ of the MTT/CCM solution $(5 \mathrm{mg} / \mathrm{mL})$ into each well. Supernatants were decanted, added with $100 \mu \mathrm{L}$ of DMSO, and incubated for $1 \mathrm{~h}\left(37^{\circ} \mathrm{C}\right)$. The absorbance was read using a spectrophotometer (Bio-Tek $\mu$ Quant) at a wavelength of $570 / 690 \mathrm{~nm}$. The percentage of viable cells was measured and a dose-response curve was generated from which the $\mathrm{IC}_{50}$ value was extrapolated.

For further analysis, the cells were exposed to sub and overt $\mathrm{IC}_{50}$ concentrations for a dose-dependent study of the novel compound. All these experiments were performed thrice independently in triplicate.

4.8. ATP Assay. HepG 2 cells $(20,000$ cells) were seeded into each well of the 96 well-plate along with $20 \mu \mathrm{L}$ CellTire Glo reagent (Promega, Madison, USA) and incubated in the 


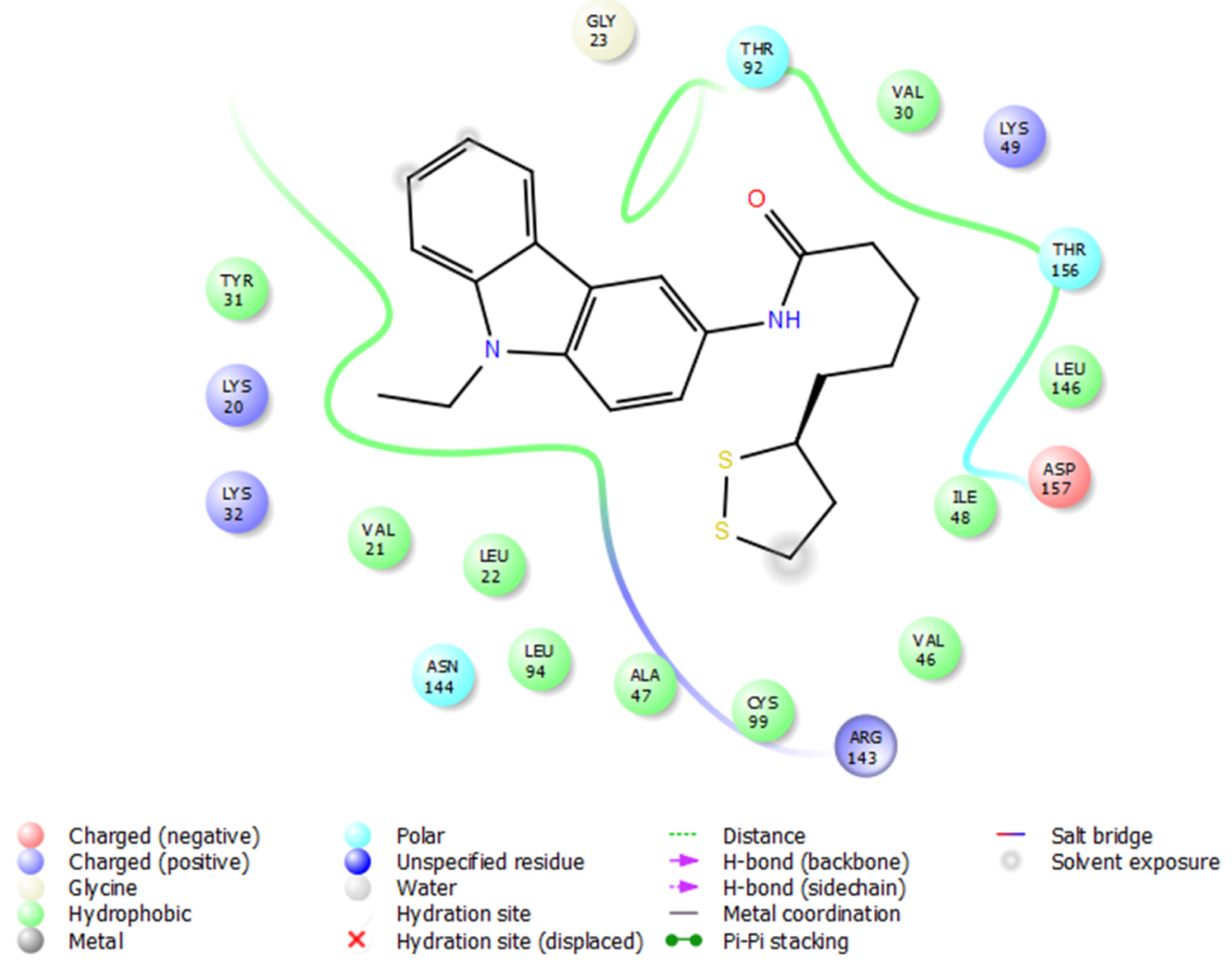

Figure 16. Interaction pattern of CT with the EGFR in molecular docking.

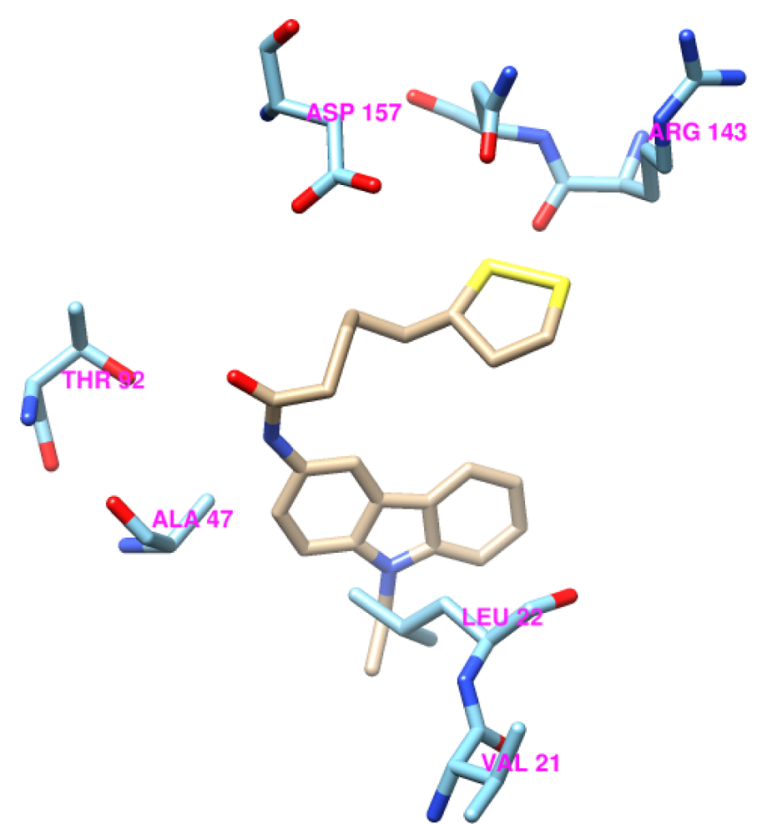

Figure 17. Binding landscape of $\mathrm{CT}$ inside the ligand binding site of the EGFR in molecular docking.

dark for $30 \mathrm{~min}$ at RT. The luminescent signal was then read using a Modulus microplate luminometer (Turner Biosystems, Sunnyvale, USA). The strength of the signal corresponds to the concentration of intracellular ATP. The results were mentioned in mean relative light units (RLU). All these experiments were repeated thrice in triplicate.

4.9. Caspase Assay. The Caspase Glo 8, 9 and 3/7 Assay kits (Promega, Madison, USA) were used to detect caspase
Table 2. Energy Components of the CT-EGFR Complex Calculated using Molecular Dynamics Simulations

$\begin{array}{lccc}\text { energy component } & \text { average } & \text { std. dev. } & \text { std. err. of mean } \\ \text { VDWAALS } & -45.3100 & 3.8646 & 0.3865 \\ \text { EEL } & -15.7461 & 7.0271 & 0.7027 \\ \text { EGB } & 27.0712 & 4.9542 & 0.4954 \\ \text { ESURF } & -5.8744 & 0.3937 & 0.0394 \\ \text { DELTA G gas } & -61.0561 & 9.8450 & 0.9845 \\ \text { DELTA G solv } & 21.1968 & 4.6821 & 0.4682 \\ \text { DELTA TOTAL } & -39.8593 & 6.1557 & 0.6156\end{array}$

activity. The same procedure was followed for the listed caspases: treated and untreated cells $(20,000$ cells $)$ were seeded into each well of the 96 well-plate along with $20 \mu \mathrm{L}$ of the Caspase Glo reagent (prepared as per the instruction manual) and incubated in the dark for $30 \mathrm{~min}$ at RT. The luminescence was detected and quantified using a Modulus microplate luminometer (Turner Biosystems, Sunnyvale, USA). The data were represented as mean RLU.

4.10. LDH Assay. The LDH cytotoxicity detection kit (Roche, Mannheim, Germany) was employed to determine cell death that occurred through membrane damage. Briefly, the supernatants $(100 \mu \mathrm{L})$ of control and treated cells were added to the wells of 96-well microtiter plate followed by the substrate mixture and left for $25 \mathrm{~min}$ at $\mathrm{RT}$, for the reaction to occur. Here, the substrate mixture has a catalyst (diaphorase/ $\mathrm{NAD}+$ ) and dye (INT/sodium lactate). Optical density was recorded spectrophotometrically at $500 \mathrm{~nm}$ (Bio-Tek uQuant). The results are expressed in mean \pm standard deviation (SD) of optical density. All these experiments were repeated thrice in triplicate. 

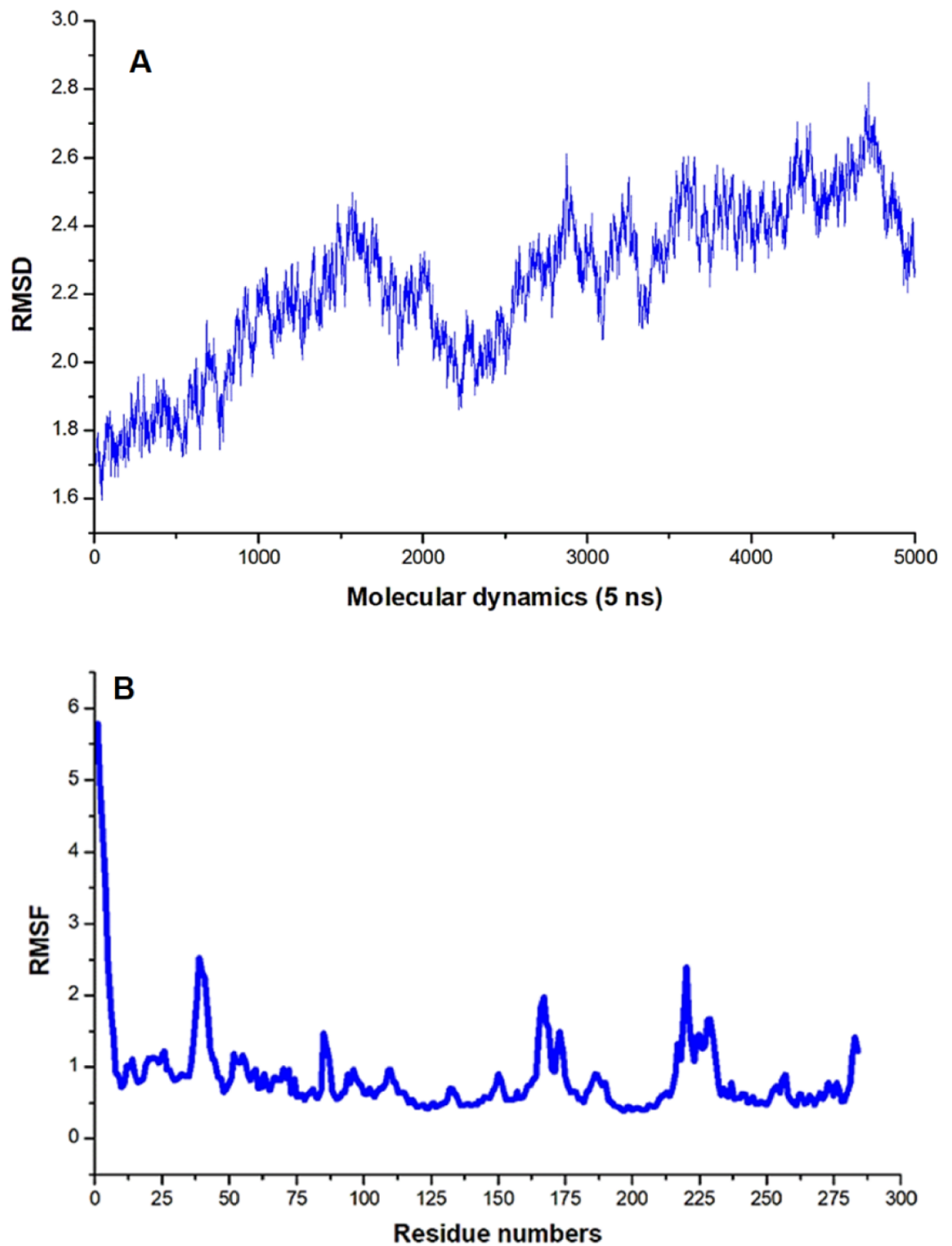

Figure 18. (A) RMSD and (B) RMSF plots of the CT with the EGFR complex obtained using molecular dynamics simulations.

\section{MOLECULAR DOCKING}

Molecular docking is used as a tool to view the interaction/ selectivity of a ligand to the active site pocket of protein. ${ }^{15}$ The 3D structure of EGFR (PDB code:6JXT) was acquired from the protein data bank. ${ }^{16}$ The structure of carbazole, LA, and CT was built using ChemDraw software. To optimize the geometry of the ligand, the MM2 force field was employed. ${ }^{17}$ The docking was carried out to study the interactions and the binding affinity of barbazole, LA, and CT with the EGFR. A grid box with a spacing of $1 \AA$ and a size of $15 \times 15 \times 15$ pointing in $x, y$, and $z$ directions was defined at the proximity of the bound ligand in the EGFR using the standard protocol. ${ }^{18,19}$ Then, the molecules were docked using AutoDockVina ${ }^{17}$ with standard docking parameters. The Lamarckian Genetic Algorithm was used as the search algorithm with standard parameter values. ${ }^{20}$ The ideal docked conformation was chosen for further investigations. Details of the docking parameters are described in our previous communications $^{21,22} \mathrm{PyMol}^{23}{ }^{23}$ Discovery Studio Visualizer, ${ }^{24}$ and $\mathrm{LigPlot}^{+25}$ were employed to visualize and analyze the structure of the docked complex.

\section{STATISTICAL ANALYSIS}

Biological experiments were conducted thrice (independently). Statistical data were evaluated by one way ANOVA and the Bonferroni test for multiple group comparisons. Results are expressed in mean $\pm \mathrm{SD}$ unless mentioned. Results with $p<$ 0.05 are statistically significant.

\section{ASSOCIATED CONTENT}

Supporting Information

The Supporting Information is available free of charge at https://pubs.acs.org/doi/10.1021/acsomega.0c04461.

IR spectrum of $\mathrm{CT},{ }^{1} \mathrm{H}$ spectrum of $\mathrm{CT}$, and ${ }^{13} \mathrm{C}$ spectrum of $\mathrm{CT}(\mathrm{PDF})$

\section{AUTHOR INFORMATION}

\section{Corresponding Authors}

Krishnan Anand - Department of Chemical Pathology, School of Pathology, Faculty of Health Sciences and National Health Laboratory Service, University of the Free State, Bloemfontein 9300, South Africa; Phone: +27632430012;

Email: organicanand@gmail.com

Muthupandian Saravanan - Department of Microbiology and Immunology, Division of Biomedical Sciences, School of 
Medicine, College of Health Sciences, Mekelle University, Mekelle 1871, Ethiopia; (1) orcid.org/0000-0002-14803555; Phone: +251344416690;

Email: saravanan.muthupandian@mu.edu.et

Anil Amichund Chuturgoon - Discipline of Medical Biochemistry, School of Laboratory Medicine and Medical Science, University of KwaZulu-Natal, Durban 4041, South Africa; (ㅇ) orcid.org/0000-0003-4649-4133;

Phone: +27312604404; Email: chutur@ukzn.ac.za

\section{Authors}

Naeem Sheik Abdul - Discipline of Medical Biochemistry, School of Laboratory Medicine and Medical Science, University of KwaZulu-Natal, Durban 4041, South Africa

Terisha Ghazi - Discipline of Medical Biochemistry, School of Laboratory Medicine and Medical Science, University of KwaZulu-Natal, Durban 4041, South Africa

Muthusamy Ramesh - Department of Pharmaceutical Analysis, Omega College of Pharmacy, Hyderabad 501 301, India

Gaurav Gupta - School of Pharmacy, Suresh Gyan Vihar University, Jaipur 302017, India

Murtaza M. Tambuwala - School of Pharmacy and Pharmaceutical Science, Ulster University, Coleraine, Northern Ireland BT52 1SA, United Kingdom; School of Biomedical Sciences, University of Ulster, Coleraine, Northern Ireland BT52 1SA, United Kingdom; () orcid.org/00000001-8499-9891

Harish Dureja - Department of Pharmaceutical Sciences, Maharshi Dayanand University, Rohtak 124001, Haryana, India

Sachin Kumar Singh - School of Pharmaceutical Sciences, Lovely Professional University, Phagwara, Punjab 144411, India

Dinesh Kumar Chellappan - School of Pharmacy, International Medical University, Kuala Lumpur 57000, Malaysia

Kamal Dua - Discipline of Pharmacy, Graduate School of Health, University of Technology Sydney, Ultimo, New South Wales 2007, Australia; Priority Research Centre for Healthy Lungs, Hunter Medical Research Institute (HMRI) \& School of Biomedical Sciences and Pharmacy, The University of Newcastle (UoN), Callaghan, New South Wales 2308, Australia; School of Pharmaceutical Sciences, Shoolini University of Biotechnology and Management Sciences, Solan 173229, India

Boomi Pandi - Department of Bioinformatics, Alagappa University, Karaikudi 630 003, India

Complete contact information is available at:

https://pubs.acs.org/10.1021/acsomega.0c04461

\section{Notes}

The authors declare no competing financial interest.

\section{ACKNOWLEDGMENTS}

The authors gratefully acknowledge the University of KwaZuluNatal and the University of the Free State, South Africa (SA) for the financial support and infrastructural facilities for this project. K.A. is grateful to National Research Foundation (NRF), SA for the research funding in the form of NRF/DSI Innovation Post-Doctoral Research Fellowship (grant no. 120677). All computational tasks were carried out using the software resources of the Center for High-
Performance Computing, Cape Town, South Africa. We are grateful to the Electron Microscope Unit, UKZN for TEM measurements and T. Govender (Department of Pharmacology, UKZN) for DLS studies.

\section{REFERENCES}

(1) Hao, G.; Sun, J.; Wei, C. Studies on interactions of carbazole derivatives with DNA, cell image, and cytotoxicity. Bioorg. Med. Chem. 2018, 26, 285-294.

(2) Yuan, Y.; Cai, T.; Xia, X.; Zhang, R.; Chiba, P.; Cai, Y. Nanoparticle delivery of anticancer drugs overcomes multidrug resistance in breast cancer. Drug Delivery 2016, 23, 3350-3357.

(3) Zhang, T.; Huang, P.; Shi, L.; Su, Y.; Zhou, L.; Zhu, X.; Yan, D. Self-Assembled Nanoparticles of Amphiphilic Twin Drug from Floxuridine and Bendamustine for Cancer Therapy. Mol. Pharm. 2015, 12, 2328-2336.

(4) Jazayeri, M. H.; Amani, H.; Pourfatollah, A. A.; Pazoki-Toroudi, H.; Sedighimoghaddam, B. Various methods of gold nanoparticles (GNPs) conjugation to antibodies. Sens Biosensing Res 2016, 9, 1722.

(5) Namiki, Y.; Takahashi, T.; Ohno, T. Gene transduction for disseminated intraperitoneal tumor using cationic liposomes containing non-histone chromatin proteins: cationic liposomal gene therapy of carcinomatosa. Gene Ther. 1998, 5, 240-246.

(6) Ding, Y.; Zhang, X.; Liu, X.; Guo, R. Adsorption Characteristics of Thionine on Gold Nanoparticles. Langmuir 2006, 22, 2292-2298.

(7) Manna, A.; Chen, P.-L.; Akiyama, H.; Wei, T.-X.; Tamada, K.; Knoll, W. Optimized Photoisomerization on Gold Nanoparticles Capped by Unsymmetrical Azobenzene Disulfides. Chem. Mater. 2003, 15, 20-28.

(8) Bistri, O.; Reinaud, O. Supramolecular control of transition metal complexes in water by a hydrophobic cavity: a bio-inspired strategy. Org. Biomol. Chem. 2015, 13, 2849-2865.

(9) Bustamante, J.; Lodge, J. K.; Marcocci, L.; Tritschler, H. J.; Packer, L.; Rihn, B. H. $\alpha$-Lipoic Acid in Liver Metabolism and Disease. Free Radic. Biol. Med. 1998, 24, 1023-1039.

(10) Meng, Q.; Hu, H.; Zhou, L.; Zhang, Y.; Yu, B.; Shen, Y.; Cong, H. Logical design and application of prodrug platforms. Polym. Chem. 2019, 10, 306-324.

(11) Huang, P.; Wang, D.; Su, Y.; Huang, W.; Zhou, Y.; Cui, D.; Zhu, X.; Yan, D. Combination of Small Molecule Prodrug and Nanodrug Delivery:Amphiphilic Drug-Drug Conjugate for Cancer Therapy. J. Am. Chem. Soc. 2014, 136, 11748-11756.

(12) Zhang, T.; Huang, P.; Shi, L.; Su, Y.; Zhou, L.; Zhu, X.; Yan, D. Self-Assembled Nanoparticles of Amphiphilic Twin Drug from Floxuridine and Bendamustine for Cancer Therapy. Mol. Pharm. 2015, 12, 2328-2336.

(13) Taylor, R. D.; MacCoss, M.; Lawson, A. D. G. Rings in Drugs. J. Med. Chem. 2014, 57, 5845-5859.

(14) Suchomel, P.; Kvitek, L.; Prucek, R.; Panacek, A.; Halder, A.; Vajda, S.; Zboril, R. Simple size-controlled synthesis of Au nanoparticles and their size-dependent catalytic activity. Sci. Rep. 2018, 8, 4589.

(15) Khan, F. I.; Wei, D.-Q.; Gu, K.-R.; Hassan, M. I.; Tabrez, S. Current updates on computer aided protein modeling and designing. Int. J. Biol. Macromol. 2016, 85, 48-62.

(16) Yan, X.-E.; Ayaz, P.; Zhu, S.-J.; Zhao, P.; Liang, L.; Zhang, C. H.; Wu, Y.-C.; Li, J.-L.; Choi, H. G.; Huang, X.; Shan, Y.; Shaw, D. E.; Yun, C.-H. Structural Basis of AZD9291 Selectivity for EGFR T790M. J. Med. Chem. 2020, 63, 8502-8511.

(17) Hanwell, M. D.; Curtis, D. E.; Lonie, D. C.; Vandermeersch, T.; Zurek, E.; Hutchison, G. R. Avogadro: an advanced semantic chemical editor, visualization, and analysis platform. J. Cheminf. 2012, 4, 17.

(18) Cosconati, S.; Forli, S.; Perryman, A. L.; Harris, R.; Goodsell, D. S.; Olson, A. J. Virtual Screening with AutoDock: Theory and Practice. Expet Opin. Drug Discov. 2010, 5, 597-607.

(19) Khan, F. I.; Nizami, B.; Anwer, R.; Gu, K.-R.; Bisetty, K.; Hassan, M. I.; Wei, D.-Q. Structure prediction and functional analyses 
of a thermostable lipase obtained from Shewanella putrefaciens. J. Biomol. Struct. Dyn. 2017, 35, 2123-2135.

(20) Trott, O.; Olson, A. J. AutoDock Vina: Improving the speed and accuracy of docking with a new scoring function, efficient optimization, and multithreading. J. Comput. Chem. 2010, 31, 455461.

(21) Khan, S.; Khan, F. I.; Mohammad, T.; Khan, P.; Hasan, G. M.; Lobb, K. A.; Islam, A.; Ahmad, F.; Imtaiyaz Hassan, M. Exploring molecular insights into the interaction mechanism of cholesterol derivatives with the Mce4A: A combined spectroscopic and molecular dynamic simulation studies. Int. J. Biol. Macromol. 2018, 111, 548560.

(22) Naz, F.; Khan, F. I.; Mohammad, T.; Khan, P.; Manzoor, S.; Hasan, G. M.; Lobb, K. A.; Luqman, S.; Islam, A.; Ahmad, F.; Hassan, M. I. Investigation of molecular mechanism of recognition between citral and MARK4: A newer therapeutic approach to attenuate cancer cell progression. Int. J. Biol. Macromol. 2018, 107, 2580-2589.

(23) Rigsby, R. E.; Parker, A. B. Using the PyMOL application to reinforce visual understanding of protein structure. Biochem. Mol. Biol. Educ. 2016, 44, 433-437.

(24) Discovery studio modeling environment. Dassault Systemes: San Diego, 2015.

(25) Laskowski, R. A.; Swindells, M. B. LigPlot+: Multiple LigandProtein Interaction Diagrams for Drug Discovery. J. Chem. Inf. Model. 2011, 51, 2778-2786.

(26) Link, S.; El-Sayed, M. A. Optical Properties and Ultrafast Dynamics of Metallic Nanocrystals. Annu. Rev. Phys. Chem. 2003, 54, 331-366.

(27) Cho, W.-S.; Thielbeer, F.; Duffin, R.; Johansson, E. M. V.; Megson, I. L.; MacNee, W.; Bradley, M.; Donaldson, K. Surface functionalization affects the zeta potential, coronal stability and membranolytic activity of polymeric nanoparticles. Nanotoxicology 2014, 8, 202-211.

(28) Arbiser, J. L.; Govindarajan, B.; Battle, T. E.; Lynch, R.; Frank, D. A.; Ushio-Fukai, M.; Perry, B. N.; Stern, D. F.; Tim Bowden, G.; Liu, A.; Klein, E.; Kolodziejski, P. J.; Tony Eissa, N.; Hossain, C. F.; Nagle, D. G. Carbazole Is a Naturally Occurring Inhibitor of Angiogenesis and Inflammation Isolated from Antipsoriatic Coal Tar. J. Invest. Dermatol. 2006, 126, 1396-1402.

(29) Moonsamy, S.; Bhakat, S.; Ramesh, M.; Soliman, M. E. S. Identification of Binding Mode and Prospective Structural Features of Novel Nef Protein Inhibitors as Potential Anti-HIV Drugs. Cell Biochem. Biophys. 2017, 75, 49-64.

(30) Ramesh, M.; Bharatam, P. V. Importance of hydrophobic parameters in identifying appropriate pose of CYP substrates in cytochromes. Eur. J. Med. Chem. 2014, 71, 15-23.

(31) Morris, G. M.; Lim-Wilby, M. Molecular docking. Methods Mol. Biol. 2008, 443, 365-382.

(32) Debnath, S.; Kanakaraju, M.; Islam, M.; Yeeravalli, R.; Sen, D.; Das, A. In silico design, synthesis and activity of potential drug-like chrysin scaffold-derived selective EGFR inhibitors as anticancer agents. Comput. Biol. Chem. 2019, 83, 107156.

(33) Cele, F. N.; Ramesh, M.; Soliman, M. E. Per-residue energy decomposition pharmacophore model to enhance virtual screening in drug discovery: a study for identification of reverse transcriptase inhibitors as potential anti-HIV agents. Drug Des., Dev. Ther. 2016, 10, 1365-1377.

(34) Pettersen, E. F.; Goddard, T. D.; Huang, C. C.; Couch, G. S.; Greenblatt, D. M.; Meng, E. C.; Ferrin, T. E. UCSF Chimera-a visualization system for exploratory research and analysis. J. Comput. Chem. 2004, 25, 1605-1612.

(35) Todorov, I. T.; Smith, W. DL_POLY_3: the CCP5 national UK code for molecular-dynamics simulations. Phil. Trans. Math. Phys. Eng. Sci. 2004, 362, 1835-1852.

(36) Jorgensen, W. L.; Chandrasekhar, J.; Madura, J. D.; Impey, R. W.; Klein, M. L. Comparison of simple potential functions for simulating liquid water. J. Phys. Chem. 1983, 79, 926-935.
(37) Berendsen, H. J. C.; Postma, J. P. M.; van Gunsteren, W. F.; DiNola, A.; Haak, J. R. Molecular dynamics with coupling to an external bath. J. Chem. Phys. 1984, 81, 3684-3690.

(38) Roe, D. R.; Cheatham, T. E. PTRAJ and CPPTRAJ: Software for Processing and Analysis of Molecular Dynamics Trajectory Data. J. Chem. Theory Comput. 2013, 9, 3084-3095. 